\title{
Association between anthraquinone laxatives and colorectal cancer: protocol for a systematic review and meta-analysis
}

\author{
Niccolò Lombardi ${ }^{1}$, Alessandra Bettiol ${ }^{1}$, Giada Crescioli ${ }^{1}$, Valentina Maggini2 ${ }^{2,3}$, Eugenia Gallo ${ }^{2,3}$, Francesco Sivelli ${ }^{3}$, \\ Francesco Sofi ${ }^{2}$, Gian Franco Gensini ${ }^{4}$, Alfredo Vannacci ${ }^{1 *}$ (D) and Fabio Firenzuoli ${ }^{3}$
}

\begin{abstract}
Introduction: Products containing anthraquinones (AQ) are mainly used as laxatives and have several biological effects. Long-term use of $\mathrm{AQ}$ laxatives is associated with an increased risk of serious adverse events (AEs), such as colorectal cancer (CRC). We will systematically synthesize the evidence on the potential association between the use of AQ laxatives and the risk of CRC.

Methods and analysis: We will search MEDLINE, Embase, Scopus, the Cochrane Library, Google Scholar, and Clinicaltrials.gov. To avoid missing any relevant studies, we will search the bibliographies of retrieved papers and recent reviews in the field. Interventions will include products containing oral AQ laxatives, in particular, those derived from rhubarb, senna, cascara, buckhorn, and aloe. Two review authors will independently screen title, abstract, and full texts and will independently extract data from included studies. The primary outcome is the number of participants diagnosed with CRC, while the secondary outcome will be cases of melanosis coli. We will also consider all other AEs reported in the included studies, in particular, intestinal bleeding, alterations of gastrointestinal motility, and potential for dependence. When possible and appropriate, for each outcome, a meta-analysis will be performed.
\end{abstract}

Discussion: This protocol is prepared in accordance with the Preferred Reporting Items for Systematic Reviews and Meta-Analyses Protocols guidelines. The protocol gives an insight into the scope and parameters for the systematic review to be carried out.

Systematic review registration: PROSPERO CRD42019125414

\section{Introduction}

Anthraquinones (AQs) are found in rhubarb root, Senna leaf and pod, Cascara, Buckhorn, and Aloe, and they are widely used in laxative preparations. AQ laxatives include physcion, chrysophanol, aloe-emodin, rhein, and sennosides. After oral ingestion, AQs are generally metabolized to active aglycones, which exert their laxative effect by damaging epithelial cells, leading directly and indirectly to changes in intestinal absorption, secretion, and motility [1]. In particular, two different mechanisms

\footnotetext{
* Correspondence: alfredo.vannacci@unifi.it

'Department of Neurosciences, Psychology, Drug Research and Child Health, Section of Pharmacology and Toxicology, Tuscan Regional Centre of Pharmacovigilance and Phytovigilance, University of Florence, Viale G. Pieraccini, 6, 50139 Florence, Italy

Full list of author information is available at the end of the article
}

of action have been proposed: (1) an effect on large intestine motility resulting in accelerated colonic transit, thus reducing fluid absorption, and (2) an effect on secretion processes resulting in enhanced fluid absorption. At a cellular level, one main target is the inhibition of the $\mathrm{Cl}^{-}$-channels across colon cells, contributing to the laxative effect [2]. Moreover, $\mathrm{Na}^{+} / \mathrm{K}^{+}$-ATPase pump is inhibited by those 1,8-dihydroxyanthrones/anthraquinones bearing an additional phenolic hydroxyl group [3].

Damaged epithelial cells can be found in the pigmented colonic mucosa, a characteristic of melanosis coli, a condition potentially related to an inappropriate use of AQ laxatives (i.e., more than 2 weeks of treatment). The question whether the melanosis coli predisposes to colorectal cancer $(\mathrm{CRC})$ is controversial [4], and the relationship between the

(c) The Author(s). 2020 Open Access This article is distributed under the terms of the Creative Commons Attribution 4.0 International License (http://creativecommons.org/licenses/by/4.0/), which permits unrestricted use, distribution, and reproduction in any medium, provided you give appropriate credit to the original author(s) and the source, provide a link to the Creative Commons license, and indicate if changes were made. The Creative Commons Public Domain Dedication waiver (http://creativecommons.org/publicdomain/zero/1.0/) applies to the data made available in this article, unless otherwise stated. 
use of AQ laxatives, melanosis coli, and CRC is still debated.

Pre-clinical studies have shown a potential role of AQ laxatives in both the initiation and promotion of tumorigenesis, and studies performed in humans have also suggested tumor promoting activities for these laxatives [5].

CRC is one of the main concerns of modern medicine, representing one of the most common types of cancer worldwide [6]. In both sexes, CRC represents the fourth type of cancer for incidence (6.1\% of the total cases) and the first one for mortality (8.2\% of the total cancer deaths).

In high-income countries, AQ laxatives are used by $20 \%$ of the population [7]. The World Health Organization (WHO) has published monographs on safety, efficacy, and quality control of Aloe, Cassia, Frangula, and Cascara for their use as medicinal plants [8]. In the monographs, it is recommended that products containing AQ glycosides should not be used for longer than 1-2 weeks, due to the possible incidence of serious AEs, such as electrolyte imbalance. Also the European Medicines Agency (EMA) recommends not using AQ laxatives (i.e., those containing rhubarb) for more than 2 weeks. Otherwise, treatment with product containing AQ laxatives should always require medical supervision [9]. In Germany, the Federal Institute for Medicines and Medical Devices has recommended not to use AQ laxatives for prolonged periods [10]. Although research to date has shown that long-term use of AQs does not necessarily lead to serious adverse events (AEs), it may be prudent to use such products only for short-term relief of constipation (e.g., potential for dependence, drug-drug interactions), in particular when they are used as a self-medication treatment [11].

Recently, the European Food Safety Authority (EFSA) provided a scientific opinion on the safety of AQ derivatives [12]. EFSA's document was triggered by concerns about the possible harmful effects associated with longterm consumption of AQ-containing preparations (i.e., food supplements used as laxatives). In particular, EFSA reviewed existing scientific evidence on the possible link between AQ products intake and adverse health effects (i.e., colorectal cancer) [12]. In their document, the panel of experts concluded that AQs should be considered as genotoxic and carcinogenic unless there are specific data to the contrary. Furthermore, they were unable to provide safety advice on the daily intake of AQ products.

Since there is no clear evidence of the potential association between the use of oral AQ laxatives and the risk of CRC, we aimed to quantify this risk by performing a systematic review and meta-analysis.

\section{Methods and analysis}

This protocol has been written according to the Preferred Reporting Items for Systematic review and Meta-Analysis
Protocols guidance $[13,14]$ and has been registered on PROSPERO (registration ID CRD42019125414).

\section{Eligibility criteria}

Studies will be selected according to the eligibility criteria outlined below.

\section{Study designs}

We will consider for inclusion both clinical trials and observational cohort studies, either prospective or retrospective. We will also include case-control studies. Observational cross-sectional studies will be excluded. Similarly, we will exclude reviews and meta-analyses, letters to the editor, case reports, case series, and expert opinions.

\section{Participants}

We will consider studies performed on subjects taking AQs as oral laxatives, excluding studies which include patients with history of any cancer. No restriction on subjects' age will be applied.

\section{Interventions}

We will consider the following plant-containing AQ laxatives:

- Senna, syn. Cassia (Cassia acutifolia, C. angustifolia)

- Frangula (Rhamnus frangula)

- Cascara (Rhamnus purshiana, Syn. Cascara sagrada)

- Rhubarb (Rheum officinale, R. palmatum)

- Aloe spp. (Aloe vera, syn. A. barbadensis, A. ferox, A. arborescens)

We will also consider all active AQ compounds, such as: physcion, chrysophanol, rhein, dantron, emodin, aloe-emodin, and senna glycosides (sennoside A and B) [15]. Additional active compounds or interventions containing AQ laxatives, not listed above and detected by screening of retrieved references or in the bibliographies of evaluated studies, will be also considered.

Studies on patients co-treated with more than one abovementioned AQ laxatives will be included as well.

\section{Comparators}

We will consider studies evaluating the effect of the above mentioned AQ laxatives compared to no treatment and/or compared to non-AQ laxatives.

\section{Outcomes}

We will include studies evaluating the primary safety outcome "CRC" and/or studies evaluating the secondary safety outcome "melanosis coli".

In studies evaluating at least one of the abovementioned safety outcomes, we will also consider the following AEs: 
1. Gastrointestinal bleeding

2. Alterations in gastrointestinal motility

3. Potential for dependence

We will also consider any other AEs experienced by treated subjects in included studies; AEs will be defined based on authors' definitions.

\section{Timing}

There will be no timing restriction. We will define a consumption of AQ laxatives less than 2 weeks as "short-term" use, while "long-term" use will be referred to as consumption longer than 2 weeks.

Regardless of the time of onset, we will include any diagnosis of CRC in patients exposed to AQ laxatives for a period exceeding 2 weeks ("long-term" use). Then we will perform a stratification based on the latency time, taking into account the clinical characteristics of each patient and evaluating the events of CRC on a case-bycase basis. For this purpose, if necessary, we will request data at the single patient level from authors of the included original studies.

\section{Setting}

There will be no restriction by type of setting.

\section{Language}

We will include articles written in any language.

\section{Information sources and search strategy}

Electronic searches will be performed in the databases MEDLINE, Embase, Scopus, the Cochrane Library, Google Scholar, and Clinicaltrials.gov.

The MEDLINE search strategy is reported below:

1. (anthraquinon*[tiab] OR anthrachinon*[tiab] OR anthraquinonoid*[tiab] OR carmine*[tiab] OR cascara*[tiab] OR emodin*[tiab] OR senna*[tiab] $O R$ cassia*[tiab] OR frangula*[tiab] OR rhamnus*[tiab] OR rheum[tiab] OR rumex*[tiab] OR rhubarb*[tiab] OR aloe*[tiab] OR sennosid*[tiab] OR physcion*[tiab] OR chrysophanol*[tiab] OR rhein $*$ tiab] OR dantron*[tiab] OR laxativ*[tiab] OR propulsiv*[tiab] OR "anthraquinones"[Mesh] OR "laxatives"[Mesh])

2. (cancer*[tiab] OR carcinom*[tiab] OR neoplas*[tiab] OR tumor[tiab] OR tumoral[tiab] OR tumorigen"[tiab] OR malignan*[tiab] OR oncogen"[tiab] OR mutagen"[tiab] OR oncolog*[tiab] OR "neoplasms"[Mesh]) AND (intestinal"[tiab] OR colon*[tiab] OR rectal*[tiab] OR colorectal*[tiab])
3. ("Hyperpigmentation"[Mesh] OR melanosis*[tiab] OR pigment"[tiab])

4. (case reports[ptyp] OR comment $[$ sb] OR editorial[ptyp] OR guideline[ptyp] OR metaanalysis[ptyp] OR practice guideline[ptyp] OR review[ptyp] OR systematic[sb])

5. (french[lang] OR spanish[lang] OR german[lang] OR chinese[lang] OR hindi[lang] OR arabic[lang] OR italian[lang] OR turkish[lang] OR swedish[lang] OR danish[lang])

6. 2 OR 3

7. 1 AND 6

8. 7 NOT 4 NOT 5

The MEDLINE search strategy will be adapted to the syntax and subject headings of the Embase, Scopus, the Cochrane Library, and Google Scholar.

Records will be retrieved on the same day from all sources.

The search strategy will be updated toward the end of the review, after being validated to ensure that the MEDLINE strategy retrieves a high proportion of eligible studies found through any means and indexed in MEDLINE.

\section{Study records \\ Data management}

Retrieved records will be managed using the software EndNote $^{\mathrm{Tm}}$.

\section{Selection process}

Two review authors will independently screen the extracted records. The two review authors will independently identify studies for inclusion by screening titles and abstracts yielded by search, eliminating those deemed irrelevant. We will retrieve full-text articles for all references that at least one of the review authors will identify for potential inclusion.

We will select studies for inclusion on the basis of review of full-text articles. We will resolve discrepancies through discussion.

Neither of the review authors will be blind to the journal titles or to the study authors or institutions.

\section{Data collection}

Two review authors will independently extract data from the included studies.

Data abstracted will include demographic information, methodology, intervention details, all reported clinically relevant conditions, and outcomes. Data will be extracted at the trial arm level. We will resolve discrepancies between authors through discussion. 


\section{Data items}

Extracted data will include the name of the study authors and year of publication, the study design and characteristics (including single or double blinding and randomization), the country in which participants were recruited, and eventual funding sources.

As for the population, we will extract the subjects' age, and clinically relevant comorbidities.

As for the intervention and the comparator, we will extract the active principle of the experimental intervention, its route of administration, the treatment dosage, and the duration of treatment.

We will extract the number of randomized participants, the number of participants included in the analysis, the number of participants with events for binary outcomes, effect size measurements (i.e., odds ratio (OR)) and variables entering the multivariable model as potential confounders, if appropriate. Whenever possible, we will use results from an intention-to-treat analysis.

\section{Outcomes and prioritization}

The primary safety outcome will be the number of subjects diagnosed with "CRC", out of the total number of treated patients.

The secondary safety outcome will be the number of cases of "melanosis coli", out of the total number of treated patients.

For all outcomes, where OR and related confidence intervals (CIs) are reported, these will be transformed to absolute numbers.

Any AE, if present, will be identified based on specific authors' definitions and will be classified using the MedDRA classification, according to preferred terms (PT) and system organ class (SOC) classification [16].

\section{Risk of bias}

Two review authors will independently assess the included studies for bias. To assess the risk of bias of included randomized controlled trials, we will follow the Cochrane Handbook for Systematic Reviews of Interventions [17]. Specifically, we will assess the risk of bias for the following domains: selection (random sequence generation; allocation concealment), performance (blinding of participants and personnel), detection (blinding of outcome), attrition (incomplete outcome data), reporting (selective reporting), and other unclear bias.

To assess the risk of bias of observational studies, we will follow the Newcastle-Ottawa Quality Assessment Scale [18]. Specifically, for included cohort studies, we will consider the following domains: selection (representativeness of the exposed cohort, selection of the nonexposed cohort, ascertainment of exposure, absence of outcome of interest at start of study), comparability, and outcome (assessment of outcome, appropriate length of follow-up, adequacy of follow-up of cohorts).

For each domain in the two tools, we will describe the procedures undertaken for each study, including verbatim quotes. A judgment as to the possible risk of bias on each domain will be made from the extracted information, rating from "low-risk" to "high-risk".

The judgements will be made independently by two review authors; disagreement will be resolved first by discussion and then by consulting a third author.

We will compute graphic representations of potential bias within included studies, using the software RevMan 5.3 (Review Manager 5.3).

\section{Data synthesis}

If studies are sufficiently homogeneous in terms of design and comparator, we will synthesize results using a meta-analysis [14].

\section{Measures of treatment effect}

All considered outcomes are based on dichotomous data. According to the assessment of statistical heterogeneity, if appropriate, for all considered outcomes, we will perform a meta-analysis using a random-effects model within a frequentist framework. We will calculate pooled ORs combining the estimates reported in each study using random-effects Mantel-Haenszel method.

For all other AEs, no quantitative synthesis will be performed, and the proportions of each reported AE will be described at study level.

\section{Unit of analysis issues}

All analysis will be conducted per trial arm, rather than at individual patient level.

\section{Dealing with missing data}

Study authors will be contacted to obtain the missing data. If missing data cannot be obtained, the study will be excluded from the related analysis.

\section{Assessment of heterogeneity}

We will evaluate the clinical heterogeneity by considering the variability in participants' features among studies and in study characteristics (study design, intervention, follow-up).

We will evaluate statistical heterogeneity across studies using the I-squared and Cochran's Q tests, and publication bias using plots of standard error against effect estimate (bias is likely to cause asymmetry in such plots) or using formal tests such as Egger one or similar.

If high levels of heterogeneity exist (I-squared $\geq 50 \%$ or $P<0.1$, we will try to explain the source of heterogeneity by conducting subgroup or sensitivity analysis. 


\section{Subgroup and sensitivity analysis}

If possible, subgroup analysis will be conducted for different AQ compounds, daily dosages, and duration of treatment (i.e., short- or long-term use).

Additional subgroup analysis will be performed, if appropriate, according to the clinical characteristics of patients in included studies.

We will conduct a sensitivity analysis including only clinical trials versus only observational studies. If possible, a second sensitivity analysis will be performed including only high-quality clinical trials.

\section{Meta-biases}

To determine whether reporting bias is present in included clinical trials, we will evaluate whether the protocol of the clinical trial has been published before recruitment of study patients. Specifically, for studies published after July 2005, we will screen the Clinical Trial Register at ClinicalTrials.gov. We will evaluate whether selective reporting of outcomes is present (outcome reporting bias). The potential for reporting bias will be evaluated using funnel plots (if $\geq 10$ studies are present).

\section{Confidence in cumulative estimate}

The quality of evidence will be judged using the Grading of Recommendations Assessment, Development and Evaluation working group (GRADE) scale, considering the domains of risk of bias, consistency, directness, precision, and publication bias. Quality will be adjudicated as high, moderate, low, or very low [17].

\section{Discussion}

To the best of our knowledge, this systematic review and meta-analysis will provide a comprehensive narrative synthesis and quantitative estimate of the risk of CRC in subjects exposed to a long-term treatment with $\mathrm{AQ}$ laxatives.

The pooled estimate will guide clinicians and policymakers in informing patients and governments about the risk associated to the use of products containing AQ laxatives. This risk could be greater for self-administered products that are easily available without a medical prescription.

Moreover, it will provide an estimate of the future global CRC burden in the context of the complementary and alternative medicine. Importantly, this systematic review will enable the identification of clinical, epidemiological, and public health gaps, thus outlining directions for further investigation.

The findings from the review will be disseminated in a peer-reviewed journal, and we will recommend or carry out research to bridge the identified gaps.

\section{Strengths and limitations}

A major strength of our study is the comprehensive review within six major databases in order to include all potential articles. Limitations include the heterogeneity in the sample size of the retrieved studies and quality of the study design. Furthermore, prospective studies with a sufficient follow-up period to observe the occurrence of CRC may be lacking.

\section{Ethics and dissemination}

There is no primary data collection involved in this study, thus research ethics approval is not required. Results will be disseminated by release of findings in a peer-reviewed scientific journal, and by abstracts and speeches at international meetings and congresses.

Provenance and peer review

Not commissioned; externally peer-reviewed

\section{Authors' contributions}

$\mathrm{NL}, \mathrm{AB}, \mathrm{GC}$, and VM contributed to concept. NL contributed to protocol registration. $\mathrm{NL}, A B, G C$, and $V M$ contributed to protocol draft. NL and $G C$ contributed to protocol editing. $A B$ contributed to data analysis plan. EG, FSi, GFG, FSo, AV, and FF contributed to protocol revising. As this is a protocol paper, the research has not yet been conducted and no data have been acquired or interpreted. All authors read and approved the final manuscript.

\section{Funding}

The authors have not received a specific grant for this research from any funding agency in the public, commercial or not-for-profit sectors.

Consent for publication

Not required

\section{Competing interests}

The authors declare that they have no competing interests.

\section{Author details}

${ }^{1}$ Department of Neurosciences, Psychology, Drug Research and Child Health, Section of Pharmacology and Toxicology, Tuscan Regional Centre of Pharmacovigilance and Phytovigilance, University of Florence, Viale G. Pieraccini, 6, 50139 Florence, Italy. ${ }^{2}$ Department of Experimental and Clinical Medicine, University of Florence, Florence, Italy. ${ }^{3}$ Research and Innovation Center in Phytotherapy and Integrated Medicine CERFIT, Referring Center for Phytotherapy, Tuscany Region, Careggi University Hospital, Florence, Italy. ${ }^{4}$ Permanent Commission for Guidelines, Tuscany Region, Florence, Italy.

Received: 1 August 2019 Accepted: 14 January 2020

Published online: 24 January 2020

\section{References}

1. Gordon M, MacDonald JK, Parker CE, et al. Osmotic and stimulant laxatives for the management of childhood constipation. Cochrane Database Syst Rev. 2016;(8):CD009118. https://doi.org/10.1002/14651858.CD009118.pub3 published Online First: Epub Date.

2. Emodin - A secondary metabolite with multiple ecological functions in higher plants. Available at: https://www.jstor.org/stable/1513810?seq= 1\#metadata_info_tab_contents. Last accessed: October 4, 2019.

3. Herbal Laxatives - Influence of anthrones-anthraquinones on energy metabolism and ion transport in a model system. Available at: https://pubs. acs.org/doi/pdf/10.1021/bk-1998-0691.ch009. Last accessed: October 4, 2019.

4. Biernacka-Wawrzonek D, Stepka M, Tomaszewska A, et al. Melanosis coli in patients with colon cancer. Przeglad gastroenterologiczny. 2017;12(1):22-7. https://doi.org/10.5114/pg.2016.64844 published Online First: Epub Date.

5. van Gorkom BA, de Vries EG, Karrenbeld A, et al. Review article: anthranoid laxatives and their potential carcinogenic effects. Alimentary pharmacology \& therapeutics. 1999;13(4):443-52. 
6. Bray F, Ferlay J, Soerjomataram I, et al. Global cancer statistics 2018: GLOBOCAN estimates of incidence and mortality worldwide for 36 cancers in 185 countries. CA Cancer J Clin. 2018;68(6):394-424. https://doi.org/10. 3322/caac.21492 published Online First: Epub Date.

7. Nusko G, Schneider B, Schneider I, et al. Anthranoid laxative use is not a risk factor for colorectal neoplasia: results of a prospective case control study. Gut. 2000;46(5):651-5.

8. World Health Organization. WHO monographs on selected medicinal plants, vol. 1. Geneva: World Health Organization; 1999.

9. European Medicines Agency. Assessment report for rhubarb (Rhei radix). Special warning/precautions for use. 2008. Available at: https://www.ema. europa.eu/en/documents/herbal-report/assessment-report-rhubarb-rheiradix_en.pdf. Last accessed: October 4, 2019.

10. Chan K, \& Lin, T. X. Treatments used in complementary and alternative medicine. Side Effects of Drugs Annual, 31 2009:745-756 doi: doi:https://doi. org/10.1016/s0378-6080(09)03148-1 [published Online First: Epub Date]|.

11. Wald A, Scarpignato C, Mueller-Lissner S, et al. A multinational survey of prevalence and patterns of laxative use among adults with self-defined constipation. Alimentary pharmacology \& therapeutics. 2008;28(7):917-30. https:/ doi.org/10.1111/j.1365-2036.2008.03806xx published Online First: Epub Date.

12. European Food Safety Authority, 2017. Safety of hydroxyanthracene derivatives for use in food. Available at: https://www.efsa.europa.eu/it/ efsajournal/pub/5090. Accessed 4 Oct 2019.

13. Moher $D$, Shamseer $L$, Clarke $M$, et al. Preferred reporting items for systematic review and meta-analysis protocols (PRISMA-P) 2015 statement. Syst Rev. 2015;4:1. https://doi.org/10.1186/2046-4053-4-1 published Online First: Epub Date.

14. Shamseer $L$, Moher D, Clarke M, et al. Preferred reporting items for systematic review and meta-analysis protocols (PRISMA-P) 2015: elaboration and explanation. BMJ. 2015;350:g7647. https://doi.org/10.1136/bmj.g7647 published Online First: Epub Date.

15. Hsu CM, Ho YP, Lien JM, et al. Endoscopic mucosal resection using a pure cut and hemoclip method for colonic nonpolypoid neoplasms. Chang Gung Med J. 2003;26(8):586-91.

16. Neubauer AS, Samari-Kermani K, Schaller U, et al. Detecting chloroquine retinopathy: Electro-oculogram versus colour vision. Br J Ophthalmol. 2003;87(7) 902-8. https://doi.org/10.1136/bjo.87.7.902 published Online First: Epub Date.

17. Higgins JP, Altman DG, Gotzsche PC, et al. The Cochrane Collaboration's tool for assessing risk of bias in randomised trials. BMJ (Clinical research ed). 2011;343:d5928. https://doi.org/10.1136/bmj.d5928 published Online First: Epub Date.

18. Sosogi A, Gao F, Tomimatsu T, et al. Non-steroidal anti-inflammatory drugs affect the methotrexate transport in IEC-6 cells. Life Sci. 2003;73(4):437-46. https:/doi. org/10.1016/S0024-3205(03)00316-3 published Online First: Epub Date.

\section{Publisher's Note}

Springer Nature remains neutral with regard to jurisdictional claims in published maps and institutional affiliations.

Ready to submit your research? Choose BMC and benefit from:

- fast, convenient online submission

- thorough peer review by experienced researchers in your field

- rapid publication on acceptance

- support for research data, including large and complex data types

- gold Open Access which fosters wider collaboration and increased citations

- maximum visibility for your research: over $100 \mathrm{M}$ website views per year

At BMC, research is always in progress.

Learn more biomedcentral.com/submissions 